\title{
Article \\ Decision Support System for Evaluating Suitable Job Applicants
}

\author{
Bogdan Walek $^{1, * \mathbb{D}}$, Ondrej Pektor $^{1}$ and Radim Farana ${ }^{2}$ \\ 1 Department of Informatics and Computers, University of Ostrava, 30. dubna 22, \\ 70103 Ostrava, Czech Republic; ondrej.pektor@osu.cz \\ 2 Department of Informatics, Mendel University in Brno, Zemědělská 1, 61300 Brno, Czech Republic; \\ radim.farana@mendelu.cz \\ * Correspondence: bogdan.walek@osu.cz
}

check for

updates

Citation: Walek, B.; Pektor, O.; Farana, R. Decision Support System for Evaluating Suitable Job Applicants. Mathematics 2021, 9, 1773. https:/ / doi.org/10.3390/math9151773

Academic Editor: Bartosz Sawik

Received: 25 June 2021

Accepted: 23 July 2021

Published: 27 July 2021

Publisher's Note: MDPI stays neutral with regard to jurisdictional claims in published maps and institutional affiliations.

Copyright: (C) 2021 by the authors. Licensee MDPI, Basel, Switzerland. This article is an open access article distributed under the terms and conditions of the Creative Commons Attribution (CC BY) license (https:/ / creativecommons.org/licenses/by/ $4.0 /)$.

\begin{abstract}
This paper describes a novel approach in the area of evaluating suitable job applicants for various job positions, and specifies typical areas of requirement and their usage. Requirements for this decision-support system are defined in order to be used in middle-size companies. Suitable tools chosen were fuzzy expert systems, primarily the inference system Takagi-Sugeno type, which were then supplied with implementation of methods of variant multi-criteria analysis. The resulting system is a variable tool with the possibility to simply set the importance of individual selection criteria so that it can be used in various situations, primarily in repeated selection procedures for similar job positions. A strong emphasis is devoted to the explanatory module, which enables the results of the expert system to be used easily. Verification of the system on real data in cooperation with a collaborating company has proved that the system is easily usable.
\end{abstract}

Keywords: decision support system; HR manager; competency model; fuzzy expert system; TakagiSugeno system; multi-criteria analysis

\section{Introduction}

Currently, the issue of human resources management (HR management) is very topical, primarily from the point of view of searching and selecting job applicants [1-5].

Every company or organization that employs staff sometimes searches for new employees. Recruitment of new employees and selection of suitable applicants for a given job position is often quite a complicated process because there are a number of requirements for hard and soft skills of the applicants as well as for their work experience. However, success of every company substantially depends on the quality and correct competencies of its employees [6]. In certain job areas, there is a significant lack of suitable workforce. In such a case, the preparations and implementation of a selection procedure entail much higher requirements.

The recruitment process of new employees, including implementation of a selection procedure, is usually solved by an HR manager or HR department. In case there is no HR manager in a company (usually small ones), the selection procedure is realized by the director or the owner of the company.

Therefore, there are a few starting points which are essential for our area of interest:

- Every company or organization sometimes searches for new employees.

- It is important to select the most suitable job applicant from all job applicants registered for a specific job position.

- Evaluation of soft skills, hard skills and work experience is needed for correct evaluation of job applicant's suitability.

From a scientific point of view, there are reasons to propose a decision support system for evaluating suitable job applicants: 
- There are several methods for calculating output based on various input parameters, different range of their values and importance of various criteria-for instance, multicriteria analysis.

- The expert system containing IF-THEN rules is a suitable tool for evaluation the suitability degree of job applicants.

- The decision support system is a suitable auxiliary tool for the HR manager who makes the final selection of the most suitable job applicant.

We expect, that proposed decision support system will be practical usable, variable and applicable for different types of job position.

The paper is organized as follows. Section 2 Current State describes current approaches and systems for evaluation of suitable job applicants, current approaches in the area of jobmatching and software tools which serve as a software support of the recruitment process. Section 3 Motivation describes the main motivations of our work. Section 4 Methodology, Model of the System describes a methodology of this research and a model of the proposed decision support system for evaluating suitable job applicants. In Section 5 Verification, the verification of the proposed system on real data in cooperation with a collaborating company is shown. Section 6 provides a discussion of the obtained results and finally, in the Conclusion, we summarize the proposed approach and decision support system and outline the goals of further research.

\section{Current State}

\subsection{Current Approaches and Systems for Evaluation of Suitable Job Applicants}

Currently, there are several approaches and systems for evaluation of suitable job applicants. Patent in [7] describes a general electronic system for evaluation of suitability of applicants for a given job position. The authors in paper [8] propose a fuzzy expert system for online recruitment of job applicants for a given job position. The input into such a system is the applicant's profile and relevant requirements for a given job position (acquired from the database of free job positions). Based on those inputs, the fuzzy expert system evaluates applicants' suitability for the job position and the information on evaluated applicants is displayed to the user. The next paper [9] describes proposed fuzzy models for HR management. The proposed models enable to evaluate applicants' suitability and to determine the degree of similarity with a so-called "ideal applicant". The authors of paper [10] describe a data mining approach based on the Rough Set Theory which enables them to find and analyze relevant data for the selection of suitable employees. The approach takes advantage of adaptive rules to select the most suitable applicants for a given job position. Another paper [11] describes a data mining framework based on a decision tree and associative rules in order to generate rules usable for the selection of suitable employees.

Authors Golec and Kahya [12] propose the selection of suitable job applicants and their evaluation using a competency-based fuzzy model, which comprises a hierarchical structure of qualities and competencies required for a given job position.

Recently, there have appeared results of work based on collaborative filtering for the automated recommendation of job positions $[13,14]$.

In addition, works researching the successfulness of decision-making systems working with incomplete or inaccurate information have been published [15], although they do not use tools of fuzzy logic, which is very suitable for such work, and their creation is possible in unusual environments, such as LabView [16].

An approach usable in applications of multi-criteria decision analysis under fuzzy environment [17] is also interesting to use in fuzzy expert systems. In the area of decision trees, there have been numerous publications published as well. An interesting paper is [18], where the authors propose a framework to evaluate a system for decision support. 


\subsection{Current Approaches in the Area of Job-Matching}

In paper [19] the author describes an expert system serving to evaluate the suitability of individual unemployed for particular job positions within so-called job-matching, i.e., matching suitable unemployed with a particular job position.

Another system is called RésuMatcher and it is closely described in [20]. It concerns a resumé (or CV) matching system which intelligently extracts work experience of the applicant directly from their CV. The same method is used to extract competencies and requirements of the job positions which the applicant applies for. The system then matches relevant job positions corresponding to the applicant's qualities and experience.

In another study [21] the authors analyze data which are being used currently for job matching. During the analysis, the key factors are identified to subsequently improve the job matching. This work then contributes to quality improvement of current approaches to job matching.

Paper [22] proposes an approach of knowledge extraction from existing online web portals offering free job positions. The authors divide the requirements stated in the description of job positions into required and desired. Moreover, they propose an approach based on knowledge extraction for suitable matching of applicants for job positions with free job positions.

Paper [23] proposes a decision-support system for evaluation of job applicants using an ideal description of a so-called job position profile. The final system then generates a report including a comparison of a candidate's profile match with the job position profile.

Certain works use algorithms from graph theory for optimal pairing of workers and job positions [24] or multi-agent systems [25] to model defined areas of the labor market.

\subsection{Software Tools}

Currently, there are several licensed or open-source systems which serve as a software support of the recruitment process. Among these open-source software systems belong, for example, OpenCats, OrangeHRM, and licensed systems, for example, WebHR or Jira. These systems primarily serve to register applicants for a job position and their qualities. Only system WebHR contains a module for easy evaluation of applicants' qualities. This evaluation is based on assigning a point evaluation (values 0-10) by an HR manager for individual qualities of a particular applicant and thus it plays only a role of a simple tool for storing point evaluation of applicant's qualities.

\section{Motivation}

The main motivation of our work is the creation of a novel approach for the evaluation of suitable job applicants for a job position. Part of our system is a decision-support system based on using a fuzzy expert system with an inference mechanism of Takagi-Sugeno type combined with a multi-criteria analysis of variants in order to find out the possibilities of further development of evaluating methods. The main aspects of our proposed solution are:

Creation of a system enabling a job position to be modelled not only using a set of qualities to be met by a job applicant, but to define their importance as well.

An important part of the system is using information on an applicant's soft skills so that the system must combine approaches of quantitative research with methods typical for qualitative research. This results in dividing the requirements into three typical quality groups which define their expectations in the area of: hard skills, soft skills, previous experience. Individual areas, however, must be open to a change in the set of evaluation criteria.

Future development of the system requires the option to select the evaluation method, its parametrization respectively, by defining the importance of individual evaluation criteria.

This brings up an issue of broad variability of the system as the requirements on the applicants change not only according to employer domain, but in time as well, which is valid for the same job position.

An important element of the system must be the explanatory mechanism, which will make clear which criteria, or areas of criteria, contribute to the evaluation of an applicant. 
This can be used by the future employer to deploy the worker on a correct job position. Meanwhile, potential mistakes in the setting of importance of individual criteria can also be revealed.

From the point of view of an HR officer, it is important to have a possibility to store information on various types of applicants' qualities (hard skills, soft skills, experience). Next, it is important to be able to return to already performed selection procedures and to create a selection procedure with similar or the same parameters as the existing ones. The possibility to easily copy already created job positions and insert them into new selection procedures is also important, as lots of selection procedures for job positions repeat or are of a very similar nature.

The created system must be then well arranged and easily understandable so that even small companies, which do not dispose of HR professionals, could use it.

\section{Methodology Model of the System}

This chapter will describe a model of the proposed system for decision support for evaluation of suitable job applicants. Our proposed system is based on a competency model, therefore let us explain the term competency model.

A competency model is a framework of qualities and skills which must be met by a competent employee for a given job position. These models are directly interconnected with company strategy. Selected qualities should help its completion. A well-defined company strategy and created competency models enable creation of a complex picture of effective staff composition of the company [26-28].

Types of qualities in a competency model are not strictly given. Various purposes and job positions require the use of various groups of qualities and skills. Generally, the following can be used:

- Hard skills;

- Soft skills;

- Length and scope of experience;

- Personal characteristics.

In this paper, we will work with the types of qualities depicted in Figure 1.

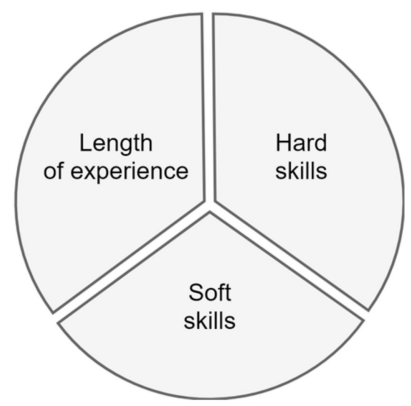

Figure 1. Used types of qualities and skills.

Figure 2 depicts a block scheme of the proposed system.

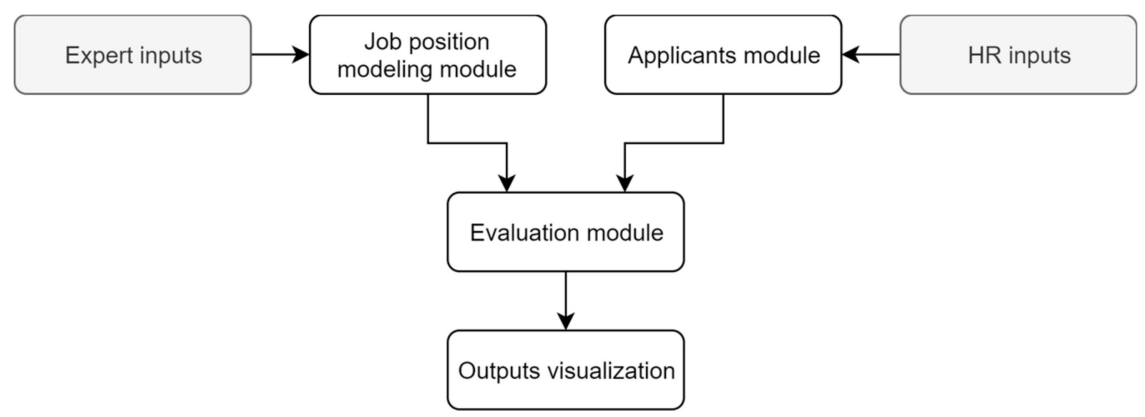

Figure 2. A block scheme of the proposed system. 
The following sub-chapters will focus on a description of individual parts of the proposed system.

\subsection{Module Job Position}

The first module of the proposed system includes the created job positions for the applicants. The process of modelling a job position is typically performed by an HR manager, who fills in the following types of input data for each job position:

- Hard skills;

- Soft skills;

- Years of experience.

The HR manager also creates a questionnaire including questions for a job position interview. The input data are then assigned its degree of importance and an ordinal range of linguistic expressions which will evaluate the attribute.

The following tables depict individual parts of such a created job position called Raynet SW developer:

- Hard skills-Table 1;

- Soft skills-Table 2;

- Years of experience-Table 3;

- Interview questionnaire-Table 4.

Table 1. Hard skills of job position as Raynet software (SW) developer.

\begin{tabular}{clc}
\hline Name & \multicolumn{1}{c}{ Range } & Importance \\
\hline HTML 5 & Nothing | Beginner | Apprentice | Wizard | Guru & 4 \\
CSS & Nothing | Beginner | Apprentice | Wizard | Guru & 4 \\
JavaScript & Nothing | Beginner | Apprentice | Wizard | Guru & 4 \\
Java & Nothing | Beginner | Apprentice | Wizard | Guru & 3 \\
Spring & Nothing | Beginner | Apprentice | Wizard | Guru & 2 \\
SQL & Nothing | Beginner | Apprentice | Wizard | Guru & 4 \\
Postgre & Nothing | Beginner | Apprentice | Wizard | Guru & 3 \\
Mongo & Nothing | Beginner | Apprentice | Wizard | Guru & 2 \\
Scala & Nothing | Beginner | Apprentice | Wizard | Guru & 1 \\
Vagrant 1 & Nothing | Beginner | Apprentice | Wizard | Guru & 1 \\
\hline
\end{tabular}

Table 2. Soft skills of job position as Raynet SW developer.

\begin{tabular}{ccc}
\hline Name & Range & Importance \\
\hline Willingness to develop & Unknown | Weak | OK | Excellent & 4 \\
Sense for detail & Unknown | Weak | OK | Excellent & 3 \\
Natural communication & Unknown | Weak | OK | Excellent & 2 \\
Raynet blood group & Nothing | Beginner | Apprentice | Wizard | Guru & 5 \\
\hline
\end{tabular}

Table 3. Years of experience of job position as Raynet SW developer.

\begin{tabular}{ccc}
\hline Name & Range & Importance \\
\hline HTML & 3 & 3 \\
CSS & 3 & 3 \\
Java & 2 & 2 \\
Spring & 1 & 1 \\
\hline
\end{tabular}

Table 4. Interview questionnaire of job position as Raynet SW developer.

\begin{tabular}{ccc}
\hline Name & Range & Importance \\
\hline Satisfaction with applicant's oral expressing & No | Small | Medium | High & 3 \\
Test assessment & Nothing | Beginner | Apprentice | Wizard | Guru & 4 \\
Overall impression & No | Small | Medium | High & 2 \\
\hline
\end{tabular}


The first column of the table states the names of individual required hard skills of the applicant. The second column states the range, which can be different for each hard skill (particular value of the range is selected by the HR manager when completing applicant's data). The third column then represents the importance of each hard skill for later evaluation. The importance is stored as number $1-5$, where 1 means the lowest importance whereas 5 is the highest importance. The table shows that there is an emphasis on the knowledge in the area of web technologies and SQL (as they have almost the highest importance in this table-value 4), other hard skills have a lower importance.

The first column of the table, similar to hard skills, states individual soft skills using columns name, range importance. The highest importance is the soft skills called Raynet blood group, which represents applicant's match with the company's culture. On the other hand, the lowest importance is laid on the soft skill called Natural communication.

The first column of the table states the name of the given experience, then the optimal years of experience required for the job position, followed by their importance. The column Optimum years of experience means the optimal length of experience for the given experience. These data were incorporated as certain skills require more years of experience than others, where it could be rather counterproductive. For instance, the position Programmer can take advantage of five-year experience rather than having 20-year experience by which there is a high possibility of the programmer having deep-rooted procedures and habits that are difficult to change. It can mean that the programmer will not be flexible enough to gain new knowledge.

The first column of the table states the name of the area which is evaluated in the interview with the applicant. The second column states the range and the third one the importance of the given area in the interview. The table shows that the job position Raynet SW developer requires the test assessment most, i.e., applicant's practical experience.

\subsection{Module Applicants}

The next module Applicants is primarily for the HR manager, who fills in the system data on individual applicants and the results of their interview.

The input of the data in this module consists of two phases:

- Filling in of all types of information based on applicant's CV;

- Completion of information about the applicant based on the interview.

The module Applicants then includes all applicants divided according to job positions they apply for. The HR manager then fills in data on all applicants for the given job position in the two phases described above.

For clarity, we state the filled-in data of a selected applicant for the job position Raynet SW developer in Table 5. The table includes all filled-in data corresponding with the structure of Tables 1-4. Individual records state the selected value of the range.

Table 5. Interview questionnaire of job position as Raynet SW developer.

\begin{tabular}{ccc}
\hline Name & Range & Importance \\
\hline Hard skills & Guru & 4 \\
HTML 5 & Guru & 4 \\
CSS & Guru & 4 \\
JavaScript & Apprentice & 2 \\
Java & Beginner & 1 \\
Spring & Wizard & 3 \\
SQL & Beginner & 1 \\
Postgre & Nothing & 0 \\
Mongo & Beginner & 1 \\
Scala & Nothing & 0 \\
\hline
\end{tabular}


Table 5. Cont.

\begin{tabular}{ccc}
\hline Name & Range & Importance \\
\hline Soft skills & Ok & 2 \\
Willingness to develop & Ok & 2 \\
Sense for detail & Excellent & 3 \\
Natural communication & Beginner & 1 \\
Raynet blood group & & \\
Years of experience & 6 & 6 \\
HTML & 6 & 6 \\
CSS & 2 & 1 \\
Java & 1 & 2 \\
Spring & & 3 \\
Interview & Medium & 2 \\
Sest assessment & Wizard & 2 \\
Overall impression & Medium & \\
\hline Satisfaction with applicant's oral expressing & Medium & \\
\hline
\end{tabular}

The table shows that the applicant masters some important hard skills very well, but some less important ones (database technologies) display minimal knowledge.

\subsection{Module Evaluation}

The first level of evaluation in this system consists in a multi-criteria analysis. This method is convenient for our purposes due to its predefined number of inputs-each job position can have a different number of criteria necessary to evaluate. In the case of using a certain type of an expert system (e.g., Mamdani), the knowledge base would have to be created for each position individually meaning that the number of rules would be huge and their consistency unbearable in the case of a higher number of criteria.

Methods of multi-criteria analysis are a subject of numerous research works, including comparative and overview studies, such as [29-31]. A number of renowned works focuses on the area of economic and social applicants, such as [32,33].

Therefore, a multi-criteria analysis offers a universal tool for the evaluation calculation of a group of criteria without prior knowledge of their exact number. This calculation takes into account particular applicant's evaluation in such criteria as well as their importance.

\section{Evaluation of Applicants' Qualities Using Multi-Criteria Analysis}

In this step, each applicant's qualities are evaluated using a multi-criteria analysis. The step is divided into several sub-steps, which are described individually.

Determination of ranges. Each criterion which is used to evaluate applicants must have a range of vague expressions which the criterion can reach. The implemented system will then have predefined general ranges which enable it to be supplemented with ranges defined by the user.

Each range should contain the expression "Unknown" as it will be default set and its whole number value will be 0 . Other vague expressions will correspond with higher numbers. These whole numbers will then be used for the calculation.

The only exception are criteria of "length of experience" type which then will be evaluated using a whole number which will state the real length of experience.

For instance, criterion "HTML 5" in Table 1 has a range composed of values "Nothing", "Beginner", "Apprentice", "Wizard" and "Guru". Expression "Nothing" corresponds to a number value 0 and "Guru" to value 4.

Determination of criteria importance. Concerning user friendliness of the system, determination of criteria importance will use only one to five points. These points will be of the following values in the calculation $1 / 5$ to $5 / 5$.

For instance, criterion "HTML 5" in Table 1 has set value of importance 4, which is almost the highest importance. 
Calculation of evaluation for one criterion. Calculation of evaluation for individual criteria for each real applicant is performed using the method of weighted sum. This sum takes place as a product of the value which the HR officer evaluated for the given criterion and the ratio of the importance of the given criteria to the overall number of importance degree.

$$
O K_{i} U_{j}=H K_{i} U_{j} *\left(\frac{V K_{i}}{P S V}\right)
$$

where $O K_{i} U_{j}$ is evaluation of the criterion $i$ for applicant $j, H K_{i} U_{j}$ is the value of criterion $i$ for applicant $j, V K_{i}$ is the importance of criterion $i$, and $P S V$ is the overall number of the degree of importance.

For instance, for the selected applicant in Table 5, the calculation of criterion "HTML $5^{\prime \prime}$ is $O K_{1} U_{1}=4 *(4 / 5)=3.2$.

Here, the years of experience are recalculated with a value which then enters the multi-criteria analysis using the optimal years of experience in the model of the job position and the set type of such attribute behavior. The most common case is that in case the applicant's evaluation exceed the optimal years of experience, the multi-criteria analysis calculation uses the optimal years of experience. In the opposite case, it could result in distorting the whole evaluation just on the basis of one longer experience which exceeds the optimal length set in the model.

For instance, for the particular applicant in Table 5, the criterion optimum years of experience for "HTML" has the value which is set as optimal in Table 3. In this case, the value stated by the applicant (6 years) is not used, but rather the optimal years of experience for this criterion "HTML"-3 years.

Calculation of applicant's suitability based on criteria. The last step of this evaluation is the calculation of each applicant's suitability in the given category. This calculation is the sum of evaluations in all criteria for the given applicant.

$$
O U_{i}=\sum_{j=1}^{n} O K_{j} U_{i}
$$

where $O U_{i}$ is evaluation of applicant $i$ and $O K_{j} U_{i}$ is evaluation of criterion $j$ for applicant $i$.

For instance, for the particular applicant in Table 5, this resulting value for hard skills, soft skills, and years of experience is $O U_{1}=24$.

Normalization of applicant's suitability. In order to normalize an applicant's suitability in percent, so-called basic alternative, which is considered for an applicant evaluated as $100 \%$. It then concerns an ideal applicant which can be really met only very rarely. Percentage suitability of each real applicant is calculated based on this reference value.

The calculation of the base is done in such a way that there is a calculation of applicant's suitability and individual values of criteria are the maximum range values.

For instance, for the job position in Table 1, criterion "HTML" is set to "Guru", its numerical value respectively, i.e., 4 . The overall evaluation of this maximum base is $O B=35.4$.

The applicant from Table 5 then has the evaluation corresponding to $67.8 \%$ of the maximum base (ideal applicant).

Evaluation of the interview. In this step, the HR officer fills the system with an overall evaluation of the interview for a given applicant. This evaluation is, once again, undertaken using a predefined range and it has an impact on the final evaluation of the applicant.

For instance, for the particular applicant in Table 5, the interview evaluation reaches value 4.4 and the interview evaluation of the maximum base is 6.2. This means that the applicant reached the evaluation of $70.9 \%$ of the interview evaluation of the maximum base.

Final evaluation. The final evaluation consists in processing inputs from both evaluations with the ratio of their importance. Thanks to the use of a fictional best applicant, it is easy to normalize its outputs into percent or any other interval. 
For instance, for the particular applicant in Table 5, the interview evaluation reaches value 4.4 and the interview evaluation of the maximum base is 6.2. This means that the applicant reached the evaluation of $70.9 \%$ of the interview evaluation of the maximum base.

The algorithm of the final evaluation is to take into consideration the output states of both preceding multi-criteria analyses. Another thing to take into consideration is the information of which multi-criteria analysis is of a higher importance. There are job positions where applicant's impression of the HR manager is more important than the assessed qualities and in some job positions it is vice versa.

Of course, there are a number of algorithms to be used for this purpose. The simplest is the method of the weighted sum, which determines the weights according to the importance of individual inputs. More complex behavior would rather be difficult to formalize.

A more formal process of evaluation could use some type of an expert system. Various alternatives and numbers of inputs can be processed, after defuzzification, using IF-THEN rules of Mamdani type. In such a case, there would be a need for a high number of rules in order to cover all possible combinations of linguistic expressions of the input variables. This fact would mean difficult tuning of such a system and even more difficult future modifications.

Due to the aforementioned reasons, the final evaluation process uses an expert system of the Takagi-Sugeno type. Thanks to the shape of IF-THEN rules, it is possible to work only with only one input variable in the antecedent, which expresses the importance of both input values. In consequence, there are numerical values of both inputs as a sum. These values, in multiplication, are then added with coefficients which take into consideration the importance of each input.

In future, using the Takagi-Sugeno type system is very beneficial in the way that if there is a need to implement, for example, behavior in zero evaluation of one input, the output must be zero, too. It means adding one more rule into the knowledge base and adjusting the antecedent of the existing rules.

Inference fuzzy system of Takagi-Sugeno type. The first ideas if the inference fuzzy system of the Takagi-Sugeno type were set down in [34]. The characteristics of the TakagiSugeno system make it usable in various types of system [35-37].

The objective of this method is to develop an intuitively behaving evaluation system for given inputs, which would be easily adjustable to user needs. This method works in a way that based on a fuzzified value of an input variable "ratio of input importance" ("IMPORTANCE"), a linear function in the consequent of the given rule is selected.

The input variable "ratio of input importance" is fuzzified according to Figure 3 below. The first fuzzy set "first" represents a state when the most important is the first input ("QUALITIES"). In the case of set "both", both inputs are of the same importance and in the case of set "second", the most important is the second input ("INTERVIEW").

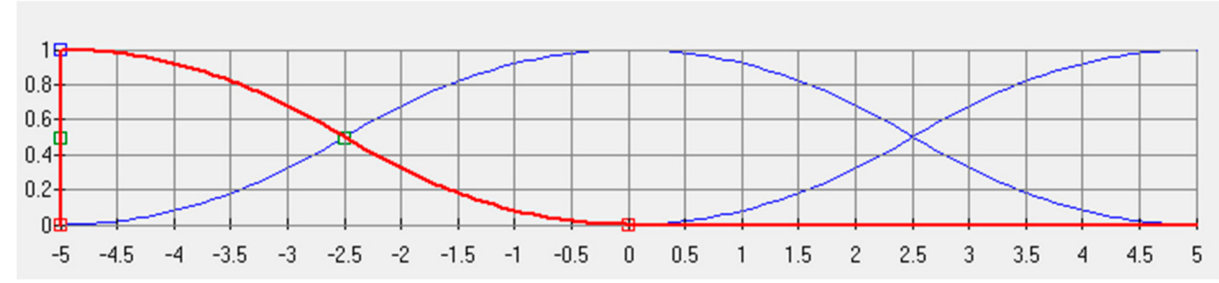

Figure 3. Membership functions of input variable ratio of input importance.

Next two input variables ("QUALITIES" and "INTERVIEW") are not a subject of defuzzification as they appear only in linear functions of IF-THEN rules consequents. These rules are provided below.

IF IMPORTANCE IS first THEN $\mathrm{y}_{1}=$ QUALITIES $\times \mathrm{C}_{\mathrm{a} 1}+$ INTERVIEW $\times \mathrm{C}_{\mathrm{b} 1}$ IF IMPORTANCE IS same THEN $\mathrm{y}_{2}=$ QUALITIES + INTERVIEW 
IF IMPORTANCE IS second THEN $\mathrm{y}_{3}=$ QUALITIES $\times \mathrm{C}_{\mathrm{a} 2}+$ INTERVIEW $\times \mathrm{C}_{\mathrm{b} 2}$

$$
\begin{gathered}
\text { For IMPORTANCE }=\text { IMPORTANCE }^{0} \\
w_{1}=\mu\left(\text { IMPORTANCE }^{0}\right) \\
\text { SUITABILITY }^{0}=\frac{w_{1} y_{1}^{0}+w_{2} y_{2}^{0}+w_{3} y_{3}^{0}}{w_{1}+w_{2}+w_{3}}
\end{gathered}
$$

The output variable "SUITABILITY" is then a result of a linear function of the selected IF-THEN rule. Coefficients $C_{a 1}, C_{b 1}, C_{a 2}$, and $C_{b 2}$ determine the influence of importance on the ratio of individual parts in the final evaluation. For the needs of this evaluation, the coefficients were determined empirically so that the influence of the importance was equally distributed to both input variables. The values of these coefficients are provided in the Table 6.

Table 6. Values of coefficients when calculating the input variable SUITABILITY.

\begin{tabular}{cc}
\hline Coefficient. & Value \\
\hline$C_{a 1}$ & 1.5 \\
$C_{b 1}$ & 0.5 \\
$C_{a 2}$ & 0.5 \\
$C_{b 2}$ & 1.5 \\
\hline
\end{tabular}

When graphically depicted, this evaluation is of a linear area shape, which rotates around its diagonal of the given space when importance is changed, see Figure 4.



Figure 4. Visualization of the final evaluation for the input importance $=0$.

\subsection{Visualization of the System Outputs}

The last step is to display system outputs in a form of evaluation of individual applicants. The outputs are displayed to the user, who is typically the HR manager who performed the selection procedure for the given job position.

Below are described real system outputs of the implemented system. Implementation is described in Section 4.5. Figure 5 depicts the final evaluation of all applicants for job position Raynet SW developer. The applicant who is described in Table 5 matches with the first row, i.e., an applicant called Applicant 2. The process of creating such a job position is closely described in Section 4.1. 


\begin{tabular}{|l|l|l|l|l|l|}
\hline First name & Last name & Evaluation of qualities & Evaluation of interview & Final evaluation & Actions \\
\hline Applicant & 2 & $68 \%$ & $71 \%$ & $69 \%$ & OSHOW EVALUATION \\
\hline Applicant & 1 & $64 \%$ & $45 \%$ & $55 \%$ & OSHOW EVALUATION \\
\hline Applicant & 3 & $37 \%$ & $19 \%$ & $28 \%$ & OSHOW EVALUATION \\
\hline
\end{tabular}

Figure 5. Final evaluation of applicants for a job position.

Figure 6 depicts the final evaluation in a graphical form using a bar chart. The first column shows the base variant, the second one Applicant 2.

\section{Comparing candidates with the base}

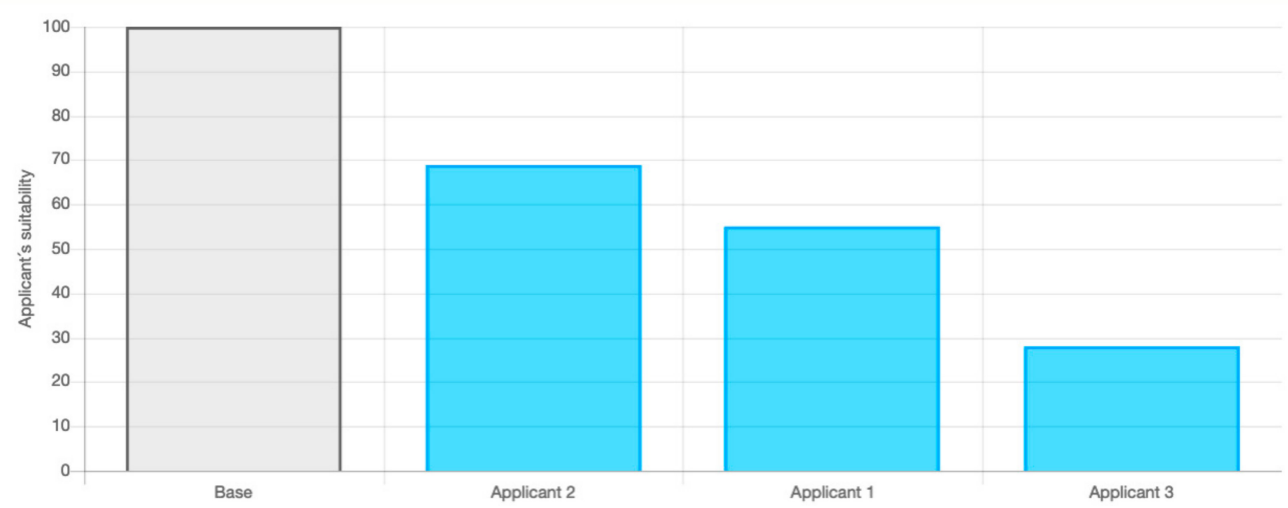

Figure 6. Chart evaluation of the applicants for a job position compared with the base variant.

In addition, it is possible to display a detailed evaluation of all applicant's qualities (hard skills, soft skills, years of experience) as well as interview evaluation for a particular applicant. The data of Applicant 2 are depicted in Figures 7 and 8.

\begin{tabular}{|c|c|c|c|c|}
\hline Attribute & Type & Verbal evaluation & Numerical evaluation & Final evaluation \\
\hline HTML5 & Hard-skills & Guru & 4 & $100 \%-(3.20 / 3.20)$ \\
\hline CSS & Hard-skills & Guru & 4 & $100 \%-(3.20 / 3.20)$ \\
\hline JavaScript & Hard-skills & Guru & 4 & $100 \%-(3.20 / 3.20)$ \\
\hline Java & Hard-skills & Apprentice & 2 & $50 \%-(1.20 / 240)$ \\
\hline Spring & Hard-skills & Begginer & 1 & $25 \%-(0.40 / 1.60)$ \\
\hline SQL & Hard-skills & Wizard & 3 & $75 \%-(2.40 / 3.20)$ \\
\hline Postgre & Hard-skills & Begginer & 1 & $25 \%-(0.60 / 240)$ \\
\hline Mongo & Hard-skills & Nothing & o & $0 \%-(0.00 / 160)$ \\
\hline Scala & Hard-skills & Begginer & 1 & $25 \%-(0.20 / 0.80)$ \\
\hline Vagrant & Hard-skills & Nothing & o & $0 \%-(0.00 / 0.80)$ \\
\hline Taste to improve & Soft-skills & Ok & 2 & $67 \%-(1.60 / 240)$ \\
\hline Attention to detail & Soft-skills & Ok & 2 & $67 \%-(1.20 / 1.80)$ \\
\hline Natural communication & Soft-skills & Great & 3 & $100 \%-(1.20 / 1.20)$ \\
\hline RAYNET blood group & Soft-skills & Weak & 1 & $33 \%-(1.00 / 3.00)$ \\
\hline HTML - optimal : 3 & Experience & 6 & 6 & $100 \%-(180 / 180)$ \\
\hline CSS - optimal : 3 & Experience & 6 & 6 & $100 \%-(1.80 / 180)$ \\
\hline Java - optimal : 2 & Experience & 2 & 2 & $100 \%-(0.80 / 0.80)$ \\
\hline Spring - optimal : 1 & Experience & 1 & 1 & $100 \%-(0.20 / 0.20)$ \\
\hline
\end{tabular}

Figure 7. Detailed evaluation of all applicant's qualities. 


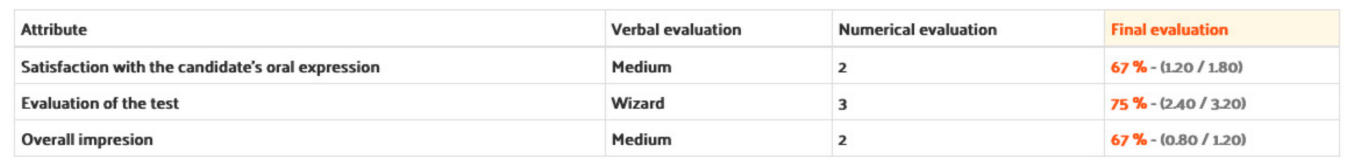

Figure 8. Detailed evaluation of questions at a job interview.

\subsection{System Implementation}

Practical verification of the system was undertaken using the implemented decisionsupport system in a form of a web application. The web applicant is called HRCalc and it contains the following main modules:

- HR officer's login;

- Management of ranges for individual job applicants' qualities (hard skills, soft skills);

- Management of selection procedures;

- Management of job positions:

- Creation of new job position;

- Copying existing job positions and their editing;

- Management of job applicants and completion of their qualities:

$\bigcirc \quad$ Possibility to add an applicant to next job position with a semi-automated copying of identical qualities from the previous positions;

- Comparison of job applicants' suitability:

- Possibility to interactively set the importance of inputs (applicant's qualities and interview);

- Evaluation of all qualities of individual job applicants.

The web application is responsive so it is possible to be used on various types of devices.

Architecture of the proposed and implemented system is depicted in Figure 9.

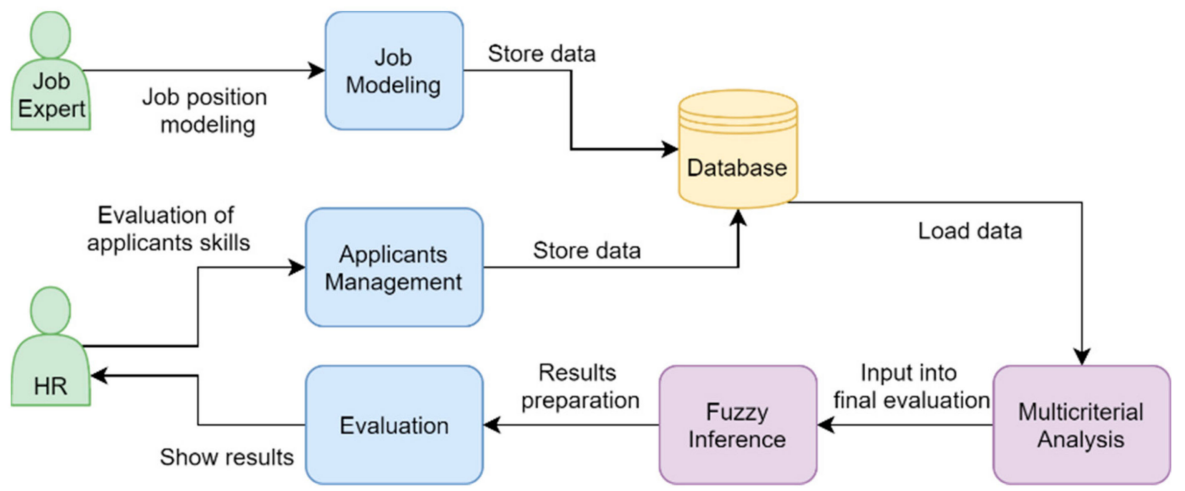

Figure 9. Architecture of the implemented system.

\section{Verification}

Our proposed decision-support system for the evaluation of suitable applicants for a job position is practically usable in various types of company which have a clear idea of the requirements of the applicants for a given job position. This system is then proposed as a support one to store information about the applicants and their qualities, and to store basic information on performed interviews for a job position.

Within our research and system proposal, we used processes valid for large companies. Based on individual needs, the system was adapted for the needs of middle-size companies as well. The verification of the system itself was performed in cooperation with an existing company which belongs to middle-sized companies.

Figure 10 depicts a process which shows individual steps from adding a new applicant into the system to the final evaluation of the suitability for a given job position. It also shows a possibility to compare the results of the selection procedure with previous selection procedures, which is an important part of the system and functionality for an HR officer. 




Figure 10. Applicant flow.

Figure 11 depicts a process of creating a new selection procedure, including adding new job positions for subsequent evaluation. During this process, the feedback mechanism is applied. After evaluating the success of the selection, the influence of individual parameters on the quality of the selected candidate is evaluated and confronted with its successful incorporation in the company. Based on this analysis, the requirements for applicants are adjusted. The system enables creation of a new selection procedure, including creation of ranges for evaluation qualities and creation of job positions which will be part of the selection procedure. An important part of the system is the possibility to copy existing job positions into a newly created selection procedure. This functionality can be used when every step of the selection procedure is repeated or to the same or similar job positions. With respect to evaluation repetition from already closed selection procedures, job positions from previous selection procedures are not transferred, but copied.

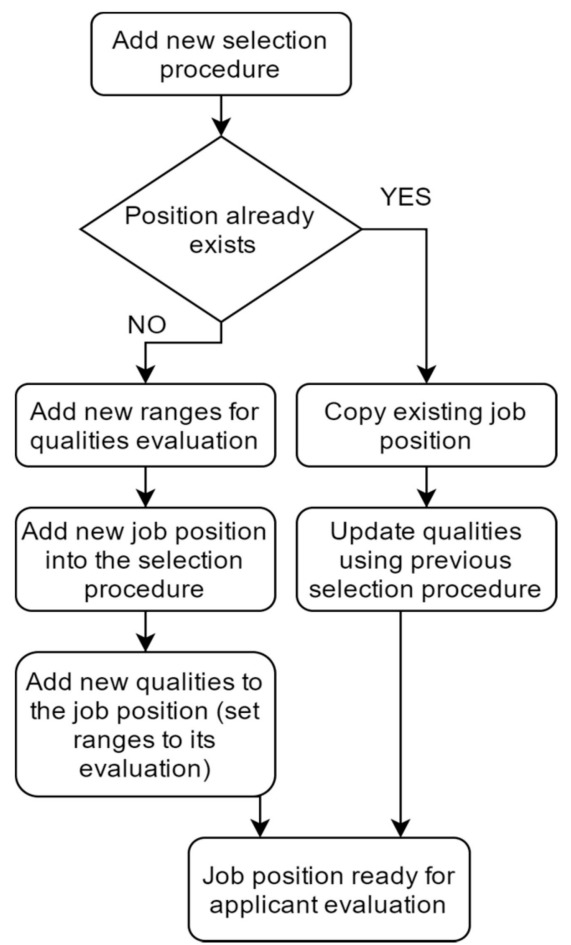

Figure 11. Modelling workflow.

This always ensures that selection procedures results can be retrospectively found out hand in hand with job positions as they were created in the past. On the other hand, copying job positions can save time when being modelled, which will be appreciated by an 
HR officer in repeated selection procedures containing the same or very similarly modelled job positions. In the case of such job positions, it is possible to add previous applicants from previous job positions into the newly created one. Of course, evaluation of new qualities which the HR officer added after the copying must be performed.

The following text will represent a practical verification of the system carried out with a cooperating company.

The verification will be demonstrated on performing two selection procedures for 2 job positions which are depicted in Table 7:

Table 7. Job positions for verification.

\begin{tabular}{cc}
\hline Name of Job Position & Number of Applicants \\
\hline Software developer & 5 \\
Business manager & 8 \\
\hline
\end{tabular}

Firstly, modelling the Software developer position will be modelled. Part of modelling a job position is defining qualities with defining ranges for individual qualities. Modelled qualities of hard skills type are depicted in Table 8 . The first column provides names of hard skills which are required for the job position by the company. The middle column provides the range used for individual hard skills. The last column provides the importance of a given hard skill. The table shows that for the given job position, the most important qualities are Windows Communication Foundation, Windows Presentation Foundation, and Team Foundation Server, for soft skills these are Activity and Harmony with a company's culture.

Table 8. Hard skills and soft skills of the modelled job position as SW developer.

\begin{tabular}{|c|c|c|}
\hline Name & Range & Importance \\
\hline \multicolumn{3}{|l|}{ Hard skills } \\
\hline Windows Communication Foundation & None | Beginner | Intermediate | Advanced | Proficiency & 5 \\
\hline Windows Presentation Foundation & None | Beginner | Intermediate | Advanced | Proficiency & 5 \\
\hline ASP.NET & None | Beginner | Intermediate | Advanced I Proficiency & 2 \\
\hline UML & None | Beginner | Intermediate | Advanced | Proficiency & 3 \\
\hline Team Foundation Server & None | Beginner | Intermediate | Advanced | Proficiency & 5 \\
\hline \multicolumn{3}{|l|}{ Soft skills } \\
\hline Desire to learn and develop & None | Small | Medium | High & 4 \\
\hline Activity & None | Small | Medium | High & 5 \\
\hline Creative approach & None | Small | Medium | High & 4 \\
\hline General communication skill & None | Small | Medium | High & 3 \\
\hline Harmony with company culture D3Soft & None | Small | Medium | High & 5 \\
\hline
\end{tabular}

Table 9 depicts modelling the years of experience which are required for the given job position. The years of experience are divided into individual categories (types), which are provided in the first column. The second column provides the required optimal years of experience. The last column provides the importance of the given type of experience.

Table 9. Years of experience modelled of the modelled job position as SW developer.

\begin{tabular}{|c|c|c|}
\hline Name & Optimum Years of Experience & Importance \\
\hline SW creation & 2 & 3 \\
\hline Experience in IT company & 2 & 3 \\
\hline Experience outside IT area & 1 & 1 \\
\hline
\end{tabular}

Table 10 shows evaluation of the skills of Applicant 4. The first column provides the name of the attribute. The second column states the evaluation and the third column then states the overall evaluation of the attribute in the ratio to the base. In the brackets is the ratio of the resulting attribute evaluation using a multi-criteria analysis to the evaluation of 
the fictional best applicant. The fictional best applicant represents a so-called basic variant or a base (a term described in more detail in Section 4.4) and it represents an ideal applicant who meets all requirements for the job position at $100 \%$.

Table 10. Evaluation of skills of Applicant 4.

\begin{tabular}{ccc}
\hline Attribute & Oral Evaluation & Overall Evaluation \\
\hline Hard skills & & \\
ASP.NET & Beginner & $25 \%-(0.4 / 1.6)$ \\
Windows Communication Foundation & Beginner & $25 \%-(1.0 / 4.0)$ \\
Windows Presentation Foundation & Beginner & $25 \%-(1.0 / 4.0)$ \\
SQL & Intermediate & $50 \%-(1.6 / 3.2)$ \\
Team Foundation Server & Intermediate & $50 \%-(1.2 / 2.4)$ \\
UML & Intermediate & $50 \%-(1.2 / 2.4)$ \\
Soft skills & & \\
Aesire to learn and develop & High & $100 \%-(2.4 / 2.4)$ \\
Activity & High & $100 \%-(3.0 / 3.0)$ \\
Creative approach & Medium & $67 \%-(1.6 / 2.4)$ \\
General communication skill & High & $100 \%-(1.8 / 1.8)$ \\
Harmony with company culture D3Soft & High & $100 \%-(3.0 / 3.0)$ \\
\hline
\end{tabular}

Table 11 depicts evaluation of years of experience for Applicant 4. The first column provides information about experience type. The second column provides the optimum length of experience required for the job position. The third column then states a real value for the applicant's experience type. The last column then states the overall evaluation the given experience type in the ratio to the base.

Table 11. Evaluation of years of experience of Applicant 4.

\begin{tabular}{ccc}
\hline Name & Optimum & Value \\
\hline Experience outside IT area & 1 & 0 \\
Experience in IT company & 2 & 0 \\
SW creation & 2 & 1 \\
\hline
\end{tabular}

Table 12 states the interview evaluation for Aplicant4. The first column provides the name of the attribute. In this case, there is only one which summarizes the overall course of the interview. Nevertheless, the system can process more attributes if the HR officer divides the interview into more parts or evaluates different attributes separately. The second column states the oral evaluation of the attribute by the HR officer. The third column then states the overall evaluation of the attribute in the ratio to the base.

Table 12. Evaluation of Applicant 4's interview.

\begin{tabular}{ccc}
\hline Attribute & Oral Evaluation & Overall Evaluation \\
\hline Overall impression & Medium & $67 \%-(2.0 / 3.0)$ \\
\hline
\end{tabular}

For illustrative purposes, Figure 12 shows a radar graph of Applicant 4 's hard skills evaluation.

The qualities in the graph are ordered clockwise from the highest acquired value to the lowest one. Such behavior is deliberate in order to maximize the surface of the graph and to eliminate solitary drops. In case particular qualities of two applicants were to be compared, the same order of the qualities would have to be kept.

Table 13 depicts the final evaluation of all applicants for a job position of software developer using an expert system of Takagi-Sugeno type. The first column states the applicant's name, the second column provides the overall evaluation of applicant's qualities (hard skills, soft skills, experience), and the third column then states the overall evaluation 
of the interview. The last column finally provides the overall evaluation by the expert system of the Takagi-Sugeno type with a different value of the inputs' degree of importance. In column $I=-5$, the qualities' evaluation is more important than interview evaluation. In column $I=0$, the qualities' evaluation is as important as interview evaluation. In column $I=-5$, the interview evaluation is more important than qualities' evaluation.



Figure 12. Hard skills of Applicant 4.

Table 13. Final evaluation of applicants' suitability for job position as SW developer.

\begin{tabular}{|c|c|c|c|c|c|}
\hline Applicant & $\begin{array}{c}\text { Inputs } \\
\text { Evaluation of Qualities }\end{array}$ & Evaluation of Interview & $\begin{array}{c}\text { Outputs } \\
I=-5\end{array}$ & $I=0$ & $I=5$ \\
\hline Applicant 4 & $57 \%$ & $67 \%$ & $60 \%$ & $62 \%$ & $63 \%$ \\
\hline Applicant 3 & $52 \%$ & $67 \%$ & $56 \%$ & $59 \%$ & $61 \%$ \\
\hline Applicant 1 & $48 \%$ & $67 \%$ & $53 \%$ & $57 \%$ & $59 \%$ \\
\hline Applicant 2 & $64 \%$ & $33 \%$ & $56 \%$ & $49 \%$ & $46 \%$ \\
\hline Applicant 5 & $37 \%$ & $33 \%$ & $36 \%$ & $35 \%$ & $35 \%$ \\
\hline
\end{tabular}

The results are also depicted in a graph in Figure 13. The first position in the graph shows the basic variant, i.e., an ideal (non-existing) applicant. The HR officer is then able to compare applicants' suitability with each other and with the ideal applicant as well. This possibility is usable when repeating similar selection procedures, where it is possible to detect an increasing or decreasing trend in the area of applicants' quality. In order to generate this chart, the degree of importance of the inputs was set to 0 .

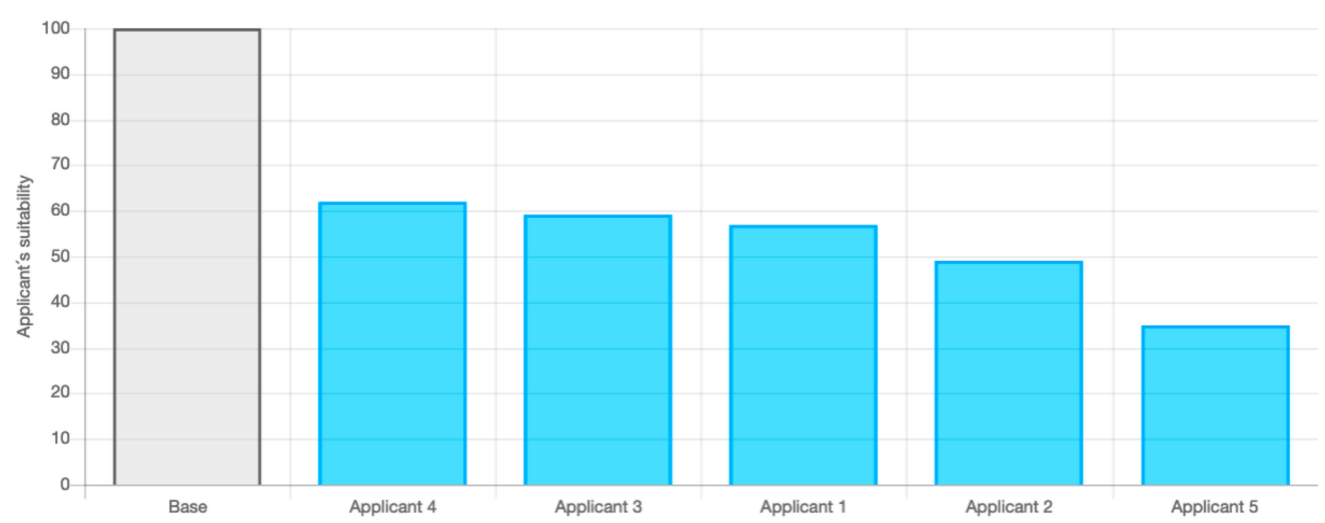

Figure 13. Final evaluation of job position SW developer with the setting of the degree of importance of the inputs to 0 .

The following part will demonstrate modelling of the second job called the Business Manager position. Firstly, it is necessary to model the job position. The modelled qualities of hard skills type are listed in Table 14. The table reveals that hard skills type qualities 
are now less numerous than for Software Developer. This fact is expected, however, as Business Manager requires more soft than hard skills.

Table 14. Hard skills and soft skills of the modelled position Business Manager.

\begin{tabular}{clc}
\hline Name & Range & Importance \\
\hline Hard skills & & \\
Communication skills & Nothing | Beginner | Intermediate | Advanced | Proficiency & 5 \\
Negotiation skills, ability to persuade & Nothing | Beginner | Intermediate | Advanced | Proficiency & 5 \\
Business drive & Nothing | Beginner | Intermediate | Advanced | Proficiency & 5 \\
Proclaimer & Nothing | Beginner | Intermediate | Advanced | Proficiency & 5 \\
General overview & Nothing | Beginner | Intermediate | Advanced | Proficiency & 5 \\
Personality of a trader & Nothing | Beginner | Intermediate | Advanced | Proficiency & 5 \\
Soft skills & & \\
Knowledge of IT technologies & Nothing | Beginner | Intermediate | Advanced | Proficiency & 3 \\
Knowledge in the field of energy & Nothing | Beginner | Intermediate | Advanced | Proficiency & 2 \\
\hline
\end{tabular}

Table 15 states a modelled length of experience, once again divided into categories (types).

Table 15. Years of experience of the modelled job position Business Manager.

\begin{tabular}{ccc}
\hline Name & Optimum Length & Importance \\
\hline Business manager in the IT company & 2 & 3 \\
Business manager in the field of energy & 2 & 3 \\
Business Manager & 4 & 5 \\
\hline
\end{tabular}

Table 16 depicts the final evaluation of all applicants' suitability for this job position using an expert system of Takagi-Sugeno type.

Table 16. Final evaluation of applicants' suitability for job position as SW developer.

\begin{tabular}{|c|c|c|c|c|c|}
\hline Applicant & $\begin{array}{c}\text { Inputs } \\
\text { Evaluation of Qualities }\end{array}$ & Evaluation of Interview & $\begin{array}{l}\text { Outputs } \\
I=-5\end{array}$ & $I=0$ & $I=5$ \\
\hline Applicant 17 & $67 \%$ & $80 \%$ & $70 \%$ & $73 \%$ & $74 \%$ \\
\hline Applicant 13 & $65 \%$ & $80 \%$ & $68 \%$ & $72 \%$ & $73 \%$ \\
\hline Applicant 18 & $65 \%$ & $60 \%$ & $64 \%$ & $63 \%$ & $62 \%$ \\
\hline Applicant 11 & $50 \%$ & $60 \%$ & $53 \%$ & $55 \%$ & $56 \%$ \\
\hline Applicant 15 & $49 \%$ & $60 \%$ & $52 \%$ & $55 \%$ & $55 \%$ \\
\hline Applicant 12 & $50 \%$ & $40 \%$ & $48 \%$ & $45 \%$ & $45 \%$ \\
\hline Applicant 14 & $26 \%$ & $20 \%$ & $25 \%$ & $23 \%$ & $23 \%$ \\
\hline Applicant 16 & $23 \%$ & $20 \%$ & $22 \%$ & $22 \%$ & $21 \%$ \\
\hline
\end{tabular}

The last three columns state the final evaluation for three reference values of the degree of importance ratio. In the evaluation, a different rule is used for each of them. The way of selecting individual rules is based on the fuzzification of the input variable IMPORTANCE and its use in the IF-THEN rules.

The results are also displayed in a graph in Figure 14. The first position in the graph represents the basic variant, i.e., an ideal (non-existing) applicant. For this chart, the value of the degree of importance of the inputs was set to 0 .

In order to calculate the values in Figure 15, the value of the degree of importance of the inputs is set to -5 .

In order to calculate the values in Figure 16, the value of the degree of importance of the inputs is set to 5 .

It is appropriate to subject the results of the multi-criteria analysis to a sensitivity analysis and to verify the influence of weight change on the overall results. Table 17 shows the results of the analysis when changing the importance criterion by 1 and 2 compared 
with the used comparison, if such a change were possible. The table also states the number of the same placed applicants for the first position.

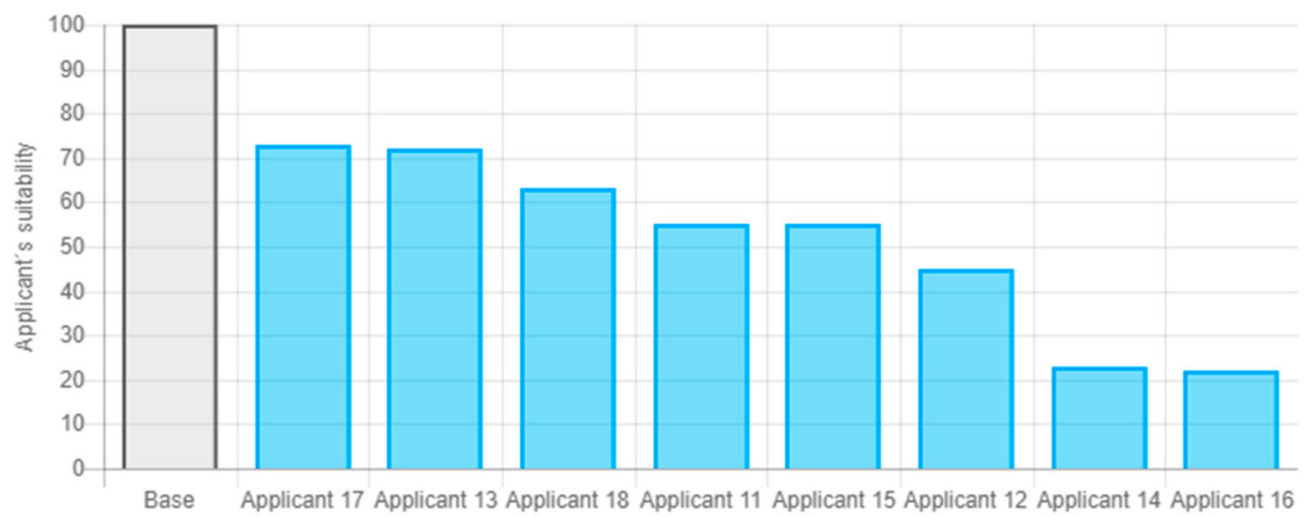

Figure 14. Final evaluation of applicants' suitability for job position as Business Manager-value of the degree of importance of the inputs is set to 0 - therefore both inputs are of the same importance.

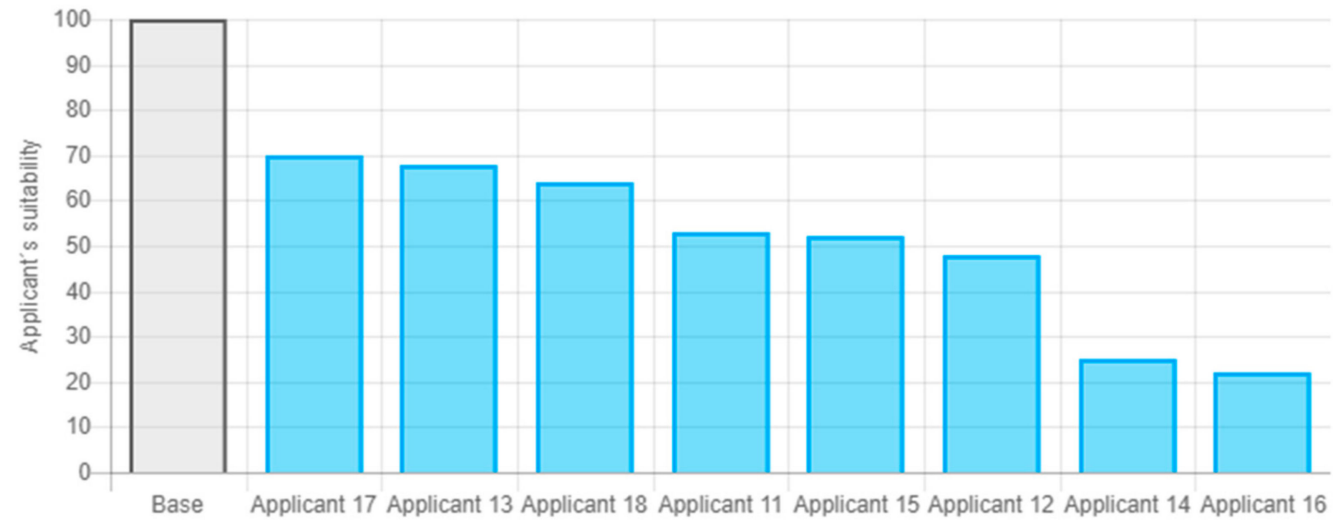

Figure 15. Final evaluation for a job position of Business Manager-value of the degree of importance of the inputs is set to -5 .

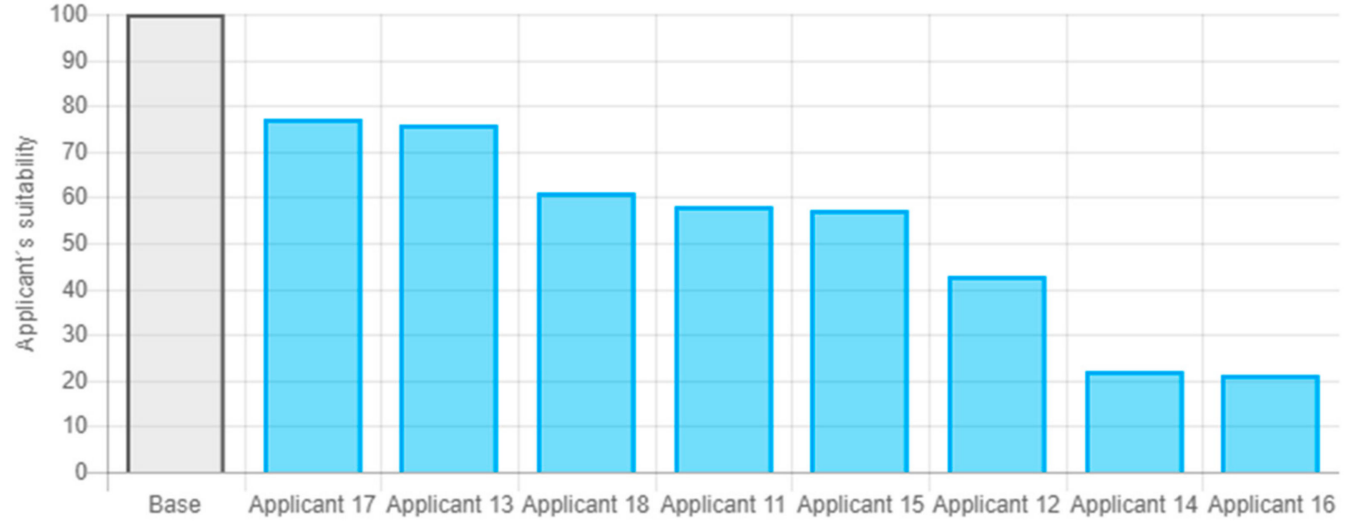

Figure 16. Final evaluation for a job position of Business Manager rule-value of the degree of importance of the inputs is set to 5 , therefore the second input is more important.

The results of the analysis show that the results are sensitive to the change of the criteria importance: client-favorable, negotiation skills, ability to persuade, and knowledge of power engineering. A specific arises in criterion Knowledge of IT technologies. The evaluation of the first two applicants differed in the maximum possible way. Therefore, increasing the importance of this criterion led to their equality until they swapped posi- 
tions. The module of the sensitivity analysis is thus important for correct interpretation of the results.

Table 17. Results of a sensitivity analysis of the multi-criteria analysis of the variants.

\begin{tabular}{cccccc}
\hline Criterion & \multicolumn{3}{c}{ Change of Weight } \\
& $-\mathbf{2}$ & $-\mathbf{1}$ & $\mathbf{0}$ & $\mathbf{1}$ & $\mathbf{2}$ \\
\hline Communication skills (Weight: 5) & 8 & 8 & 8 & - & - \\
Business drive (Weight: 5) & 8 & 8 & 8 & - & - \\
Business manager (Weight: 5) & 8 & 8 & 8 & - & - \\
Business manager in IT company (Weight: 3) & 8 & 8 & 8 & 8 & 8 \\
Business manager in power engineering (Weight: 3) & 8 & 8 & 8 & 8 & 8 \\
Business personality (Weight: 5) & 8 & 8 & 8 & - & - \\
Client-favourable (Weight: 5) & 3 & 3 & 8 & - & - \\
Negotiation skills, ability to persuade (Weight: 5) & 3 & 3 & 8 & - & - \\
General knowledge (Weight: 5) & 8 & 8 & 8 & - & - \\
Knowledge of IT technologies (Weight: 3) & 0 & 0 & 8 & 8 & 8 \\
Knowledge of power engineering (Weight: 2) & - & 8 & 8 & 4 & 4 \\
\hline
\end{tabular}

The presented results of the verification of the whole system in practice reveals that the selected procedures have been proven right. The same methodological approach could be used for a very different job position.

\section{Discussion}

\subsection{Evaluation of Applicants' Suitability for a Job Position and Their Comparison with an} Ideal Applicant

The results provided in Figures 7 and 8, and Tables pointed out the fact that applicants with better evaluation of qualities (hard skills, soft skills, experience) were also better at the interview. An interesting finding is a comparison of applicants with each other and with the ideal applicant (non-existing one). This information can be beneficial for an HR officer in the final selection of the most suitable applicant for a given job position.

\subsection{Displaying Applicant's Qualities in a Radar Graph}

Figure 6 depicts hard skills qualities of one applicant for a job position, namely Applicant 4. Thanks to this graph, it is possible to find out quickly the weaknesses and strengths of the given applicant with respect to the ideal applicant's qualities. In the case of displaying radar graphs of more applicants, it is then possible to easily compare the qualities of individual applicants.

\subsection{Variability of the Final Evaluation}

Figure 17 depicts an important system functionality, which enables an HR officer to set 3 possible situations for the final evaluation:

- Evaluation of applicant's qualities (hard skills, soft skills, experience) is of a higher importance than interview evaluation;

- Evaluation of applicant's qualities (hard skills, soft skills, experience) is of a lower importance than interview evaluation;

- Evaluation of applicant's qualities (hard skills, soft skills, experience) is of the same importance as interview evaluation.


Figure 17. Slider for variability of the final evaluation. 
Using this functionality, an HR officer can determine the importance of both main evaluation parts, which then influence the final evaluation. A premise is that this functionality will be used very frequently as an HR officer typically opens selection procedures with more important applicant's qualities for the job position (e.g., the Software Developer position) and with more important overall impression from the interview (e.g., Business manager position).

\subsection{Comparison with Other Approaches}

For the purposes of comparing our decision support system for evaluating suitable job applicants with other approaches, we selected the approaches stated in 2.1.

In paper [7], there is a description of a general electronic system for the evaluation of suitable job applicants. Similarly to our approach, this approach enables to define various types of applicant's qualities and to evaluate them. It also enables hard skills, soft skills, and a job interview to be evaluated. The result is a well-arranged table sorting the applicants according to their suitability. The difference is the use of evaluating individual qualities with values ranging 0-10 and the calculation of the applicant's final score. In contrast to this approach, the advantage of our system is the variability of the final evaluation, which enables the importance of applicant's qualities and the interview evaluation to be set, thereby influencing the final evaluation of applicants for the given job position.

In paper [8], a fuzzy expert system is proposed for online recruitment of applicants. Similarly to our approach, even this approach uses the expression "ideal applicant" and each applicant's suitability is then calculated with respect to the ideal applicant. Our advantage over this approach is the possibility to read online job advertisements containing requirements for job positions and the applicants are then evaluated for those job positions. The advantage of our approach is the possibility to evaluate applicant's qualities and job interview separately. In addition, it is the variability of the final evaluation based on the selected importance of these partial evaluations.

In paper [9], fuzzy models for human resource management are described. The proposed models enable to evaluate applicant's suitability and to determine the degree of similarity with a so-called "ideal applicant". The advantage of such created fuzzy models is the possibility to determine the degree of similarity of the given applicant with respect to the "ideal applicant". In our system, it is possible to find out the degree of similarity with the "ideal applicant" for an individual applicant's qualities using radar graphs. However, in our approach, there is no complex method to determine the overall similarity of the given applicant with respect to the "ideal applicant".

In paper [10], the authors describe a data mining approach based on the Rough Set Theory, which enables to find and analyze relevant data for the selection of suitable workers. The advantage of this approach, unlike our approach, is the data mining method to read data of the applicants and their qualities.

In paper [11], there is a description of a data mining framework based on a decision tree and associative rules to generate usable rules to select suitable workers. The approach is rather focused on a comparison of various groups of applicants (e.g., applicants with a university degree M.A, M.Sc., Ph.D., etc.) as well as on deducing findings, such as applicant's suitability, work efficiency, loyalty to the employer, behavior of the worker from a long-term perspective, etc. The approach is therefore different from ours in a way that based on the data on applicant's age, sex, marital status, education, work experience, etc., it predicts applicant's efficiency for the given job position and the level of the loyalty to the employer.

In paper [12], the authors propose a competency-based fuzzy model to select suitable applicants. It consists of a hierarchical structure of qualities and competencies necessary for a given job position. The advantage of this approach is the possibility to create a general model comprising a hierarchical structure of categories of qualities and competencies necessary for a given job position. Therefore, the model is usable in various types of companies with different requirements in the evaluation of applicants. Our approach 
also enables various applicant's qualities to be created for a given job position, but the advantage of the described approach is the creation of an arbitrary hierarchical structure of qualities and competencies necessary for a given job position. The advantage of our approach is the variability of the final evaluation based on the selected importance of the partial evaluations.

Table 18 presents the results of qualitative analysis and comparison of the proposed system with other systems for evaluating the suitability of job applicants. It is clear from the table that our system is closely focused on the area of evaluating the suitability of candidates. None of the compared systems solves this area in a more sophisticated way.

Table 18. Comparison of the proposed system with other systems.

\begin{tabular}{|c|c|c|c|c|}
\hline Attribute & HR Calc & Open CATS & Orange HRM & Web HR \\
\hline Hiring workflow & no & $\begin{array}{l}\text { elaborated in eight } \\
\text { states }\end{array}$ & $\begin{array}{l}\text { rejected, shortlisted, } \\
\text { scheduled interview, } \\
\text { accepted and not } \\
\text { accepted }\end{array}$ & $\begin{array}{l}\text { sending e-mails and } \\
\text { scheduling interviews }\end{array}$ \\
\hline $\begin{array}{l}\text { Evaluation of the } \\
\text { suitability of } \\
\text { candidates }\end{array}$ & $\begin{array}{l}\text { multicriteria analysis } \\
\text { and external system }\end{array}$ & manual number of stars & $\begin{array}{l}\text { only the candidate's } \\
\text { procedure in the } \\
\text { workflow }\end{array}$ & $\begin{array}{c}\text { filtering by multiple } \\
\text { properties in each } \\
\text { category }\end{array}$ \\
\hline Wages & none & none & none & detailed with statistics \\
\hline Time planning & none & planning job interviews & $\begin{array}{l}\text { company calendars } \\
\text { with the possibility of } \\
\text { reporting time worked }\end{array}$ & $\begin{array}{c}\text { assignment of tasks to } \\
\text { employees, including } \\
\text { missions }\end{array}$ \\
\hline Employee agenda & no & no & yes & yes \\
\hline Responsive design & yes & no & no & yes \\
\hline $\begin{array}{l}\text { Sending Emails to } \\
\text { Applicants }\end{array}$ & no & yes & no & yes \\
\hline Location of workplaces & no & no & yes & yes \\
\hline $\begin{array}{l}\text { Calendar of job } \\
\text { interviews }\end{array}$ & no & yes & no & yes \\
\hline
\end{tabular}

\section{Conclusions}

This paper presents a newly created decision-support system for evaluating suitable job applicants. The main benefits of the system are its high variability and ability to customize according to the requirements for a given job position as well as ability to react to the changes in the requirements in time. The foundation of the system is a created competency model, which divide the required applicant's qualities into hard skills, soft skills, and experience. All three groups are of a structured form, which was not common before, primarily in soft skills and experience requirements.

The created system favorably takes advantage of a fuzzy expert system of the TakagiSugeno type and combines it with multi-criteria analysis approach. The result is a robust decision-support system with a high level of variability able to perform individual selection procedures easily and effectively for various job positions as well as to encompass changing requirements for the same job position in time.

A strength of this system is its explanatory mechanism, which clearly shows individual parameters contributing to the evaluation of a given applicant, so that revealing possible mistakes in the evaluation of the importance, or another criterion, is simple.

The created system has been successfully verified in a cooperating industrial partner company during the performance of particular selecting procedures and it has proved its advantages. The result is thus a system which uses findings from large companies and, despite being developed for use in middle-size companies, it can be used easily in the environment of small companies, which do not have a professional HR officer and where the HR activities are done by the director or the owner of the company. 
Our future research will be focused on the creation of a general system for evaluation of competencies together with an automated system for finding suitable competencies for various job positions using data from job web portals with methods of data mining.

Author Contributions: Conceptualization, B.W. and R.F.; methodology, B.W., O.P., R.F.; software, O.P.; validation, B.W., O.P. and R.F.; formal analysis, B.W. and R.F.; investigation, B.W. and R.F.; resources, B.W. and O.P.; data curation, B.W., O.P. and R.F.; writing—original draft preparation, B.W. and O.P.; writing—review and editing, B.W., O.P. and R.F.; visualization, O.P.; supervision, B.W.; project administration, B.W.; funding acquisition, B.W. All authors have read and agreed to the published version of the manuscript.

Funding: This work was created during the completion of a Student Grant SGS20/PřF-MF/2021 with student participation, supported by the Czech Ministry of Education, Youth and Sports.

Conflicts of Interest: The authors declare no conflict of interest.

\section{References}

1. Schuler, R.S. Strategic human resources management: Linking the people with the strategic needs of the business. Organ. Dyn. 1992, 21, 18-32. [CrossRef]

2. Losey, M.R. Mastering the competencies of HR management. Hum. Resour. Manag. 1999, 38, 99-102. [CrossRef]

3. Undurraga, R. Who will get the job? Hiring practices and inequalities in the Chilean labour market. Bull. Lat. Am. Res. 2019, 38, 575-590. [CrossRef]

4. Harris, C.G. Finding the best job applicants for a job posting: A comparison of human resources search strategies. In Proceedings of the 2017 IEEE International Conference on Data Mining Workshops (ICDMW), New Orleans, LA, USA, 18-21 November 2017; pp. 189-194.

5. Melão, N.; Reis, J. Using Social Networks in Personnel Selection: A Survey of Human Resource Professionals. In Proceedings of the 2020 15th IEEE Iberian Conference on Information Systems and Technologies (CISTI), Seville, Spain, 24-27 June 2020; pp. 1-6.

6. Chao, Y.-P.; Lai, W.-H.; Chou, Y.-C. Investigating the relationship between competency development and organizational performance. In Proceedings of the PICMET'14 IEEE Conference: Portland International Center for Management of Engineering and Technology, Infrastructure and Service Integration, Kanazawa, Japan, 27-31 July 2014.

7. Dewar, K.L. Electronic Predication System for Assessing a Suitability of Job Applicants for an Employer. U.S. Patent 7,606,778, 20 October 2009.

8. Daramola, J.O.; Olufunke, O.O.; Musa, A.G. A fuzzy expert system (FES) tool for online personnel recruitments. Int. J. Bus. Inf. Syst. 2010, 6, 444-462. [CrossRef]

9. Canos, L.; Vicente, L. Some fuzzy models for human resource management. Int. J. Technol. Policy Manag. 2004, 4, 291-308. [CrossRef]

10. Chien, C.F.; Chen, L.F. Using rough set theory to recruit and retain high-potential talents for semiconductor manufacturing. IEEE Trans. Semicond. Manuf. 2007, 20, 528-541. [CrossRef]

11. Chien, C.F.; Chen, L.F. Data mining to improve personnel selection and enhance human capital: A case study in high-technology industry. Expert Syst. Appl. 2008, 34, 280-290. [CrossRef]

12. Golec, A.; Esra, K. A fuzzy model for competency-based employee evaluation and selection. Comput. Ind. 2007, 52, 143-161. [CrossRef]

13. Jarrett, J.; Blake, M.B. Using Collaborative Filtering to Automate Worker-Job Recommendations for Crowdsourcing Services. In Proceedings of the 2016 IEEE International Conference on Web Services, San Francisco, CA, USA, 27 June-2 July 2016 ; pp. 641-645. [CrossRef]

14. Jarrett, J.; Blake, M.B. Interoperability and Scalability for Worker-Job Matching across Crowdsourcing Platforms. In Proceedings of the 2017 IEEE 26th International Conference on Enabling Technologies: Infrastructure for Collaborative Enterprises, Poznan, Poland, 21-23 June 2017; pp. 3-8. [CrossRef]

15. Canellas, M.C.; Feigh, K.M.; Chua, Z.K. Accuracy and Effort of Decision-Making Strategies with Incomplete Information: Implications for Decision Support System Design. IEEE Trans. Hum. Mach. Syst. 2015, 45, 686-701. [CrossRef]

16. Wadgaonkar, J.; Bhole, K. Fuzzy Logic Based Decision Support System. In Proceedings of the 2016 1st IEEE India International Conference on Information Processing (IICIP), Delhi, India, 12-14 August 2016.

17. Yatsalo, B.; Korobov, A.; Martínez, L. Fuzzy multi-criteria acceptability analysis: A new approach to multi-criteria decision analysis under fuzzy environment. Expert Syst. Appl. 2017, 84, 262-271. [CrossRef]

18. Akoka, J. A framework for decision support systems evaluation. Inf. Manag. 1981, 4, 133-141. [CrossRef]

19. Drigas, A.; Kouremenos, S.; Vrettos, S.; Vrettaros, J.; Kouremenos, D. An expert system for job matching of the unemployed. Expert Syst. Appl. 2004, 26, 217-224. [CrossRef]

20. Guo, S.; Alamudun, F.; Hammond, T. RésuMatcher: A personalized résumé-job matching system. Expert Syst. Appl. 2016, 60, 169-182. [CrossRef] 
21. Kino, Y.; Kuroki, H.; Machida, T.; Furuya, N.; Takano, K. Text Analysis for Job Matching Quality Improvement. Procedia Comput. Sci. 2017, 112, 1523-1530. [CrossRef]

22. Chala, S.; Harrison, S.; Fathi, M. Knowledge extraction from online vacancies for effective job matching. In Proceedings of the 2017 IEEE 30th Canadian Conference on Electrical and Computer Engineering (CCECE), Windsor, ON, Canada, 30 April-3 May 2017.

23. Saat, N.M.; Singh, D. Assessing suitability of candidates for selection using candidates' profiling report. In Proceedings of the 2011 IEEE International Conference on Electrical Engineering and Informatics, Bandung, Indonesia, 17-19 July 2011.

24. Hao, W.; Yang, Y.; Lin, C.; Zhai, Z. QoS-aware Scheduling Algorithm Based on Complete Matching of User Jobs and Grid Services. In Proceedings of the 2006 IEEE Asia-Pacific Conference on Services Computing (APSCC'06), Guangzhou, China, 12-15 December 2006.

25. Mori, K.; Kurahashi, S. Optimizing of Support Plan for New Graduate Employment Market: Reinforcement Learning. In Proceedings of the SICE Annual Conference 2010, The Grand Hotel, Taipei, Taiwan, 18-21 August 2010; pp. $1281-1282$.

26. Campion, M.A.; Fink, A.A.; Ruggeberg, B.J.; Carr, L.; Phillips, G.M.; Odman, R.B. Doing competencies well: Best practices in competency modeling. Pers. Psychol. 2011, 64, 225-262. [CrossRef]

27. García-Barriocanal, E.; Sicilia, M.A.; Sánchez-Alonso, S. Computing with competencies: Modelling organizational capacities. Expert Syst. Appl. 2012, 39, 12310-12318. [CrossRef]

28. Shippmann, J.S.; Ash, R.A.; Batjtsta, M.; Carr, L.; Eyde, L.D.; Hesketh, B.; Kehoe, J.; Pearlman, K.; Prien, E.P.; Sanchez, J.I. The practice of competency modeling. Pers. Psychol. 2000, 53, 703-740. [CrossRef]

29. Belton, V.; Stewart, T. Multiple Criteria Decision Analysis: An Integrated Approach; Springer Science \& Business Media: Berlin/Heidelberg, Germany, 2002.

30. Zopounidis, C.; Pardalos, P.M. (Eds.) Handbook of Multicriteria Analysis; Springer Science \& Business Media: Berlin/Heidelberg, Germany, 2010; Volume 103.

31. Triantaphyllou, E. Multi-Criteria Decision Making Methods: A Comparative Study; Kluwer Academic Publishers: Boston, MA, USA, 2000.

32. Saaty, T.L.; Vargas, L.G. Decision Making in Economic, Political, Social, and Technological Environments with the Analytic Hierarchy Process; Rws Publications: Pittsburgh, PA, USA, 1994; Volume 7.

33. Ventre, A.G.; Maturo, A.; Hošková-Mayerová, Š.; Kacprzyk, J. (Eds.) Multicriteria and Multiagent Decision Making with Applications to Economics and Social Sciences; Springer: New York, NY, USA, 2013; Volume 305.

34. Takagi, T.; Sugeno, M. Fuzzy identification of systems and its applications to modeling and control. IEEE Trans. Syst. Man Cybern. 1985, 1, 116-132. [CrossRef]

35. Lohani, A.K.; Goel, N.K.; Bhatia, K.K.S. Takagi-Sugeno fuzzy inference system for modeling stage-discharge relationship. J. Hydrol. 2006, 331, 146-160. [CrossRef]

36. Xiong, L.; Shamseldin, A.Y.; O'connor, K.M. A non-linear combination of the forecasts of rainfall-runoff models by the first-order Takagi-Sugeno fuzzy system. J. Hydrol. 2001, 245, 196-217. [CrossRef]

37. Angelov, P. Evolving Takagi-Sugeno Fuzzy Systems from Streaming Data (eTS+). Evol. Intell. Syst. Methodol. Appl. 2010, 12, 21-50. 